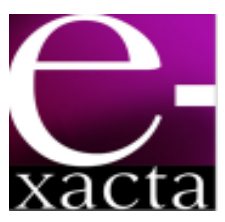

ISSN: 1984-3151

\section{ESTUDO DO EFEITO DA SECAGEM POR RADIAÇÃO ULTRAVIOLETA NAS PROPRIEDADES TECNOLÓGICAS DA FÉCULA DE MANDIOCA FERMENTADA}

\section{STUDY OF THE EFFECT OF DRYING USING ULTRAVIOLET RADIATION ON THE TECHNOLOGYCAL PROPERTIES OF THE FERMENTED CASSAVA STARCH}

\author{
A.C. S. D. V. MACHADO ${ }^{1}$; I. P. DINIZ ${ }^{2}$; M. A. V. TEIXEIRA ${ }^{3}$; V. S. BIRCHAL \\ 1 Mestre em Engenharia Química. DEQ - Escola de \\ Engenharia/UFMG, 2012. anaserraduarte@yahoo.com.br. \\ 2 Mestre em Ciência e Tecnologia de Alimentos - Universidade \\ Federal de Viçosa - UFV, 2006 - Engenheiro de Pesquisa e \\ Desenvolvimento - Chocolates Garoto S.A. \\ presotti4@yahoo.com.br. \\ 3 Doutora em Tecnologia de Alimentos. Universidade Estadual de \\ Campinas - UNICAMP, 2002. Professora do Departamento de \\ Alimentos- Escola de Farmácia/UFMG. Belo Horizonte, Minas \\ Gerais. mavtgarcia@gmail.com. \\ 4 Doutora em Engenharia Mecânica. DEMEC - Escola de Engenharia \\ da UFMG, 2003. Professora do Departamento de Engenharia \\ Química - Escola de Engenharia/UFMG. Belo Horizonte, Minas \\ Gerais. vsbirchal@gmail.com.
}

Recebido em: 10/07/2012 - Aprovado em: 14/07/2012 - Disponibilizado em: 30/07/2012

\begin{abstract}
RESUMO: O processo de produção de polvilho azedo consiste, basicamente, da fermentação natural da fécula de mandioca seguida pela secagem solar. Este processo está sujeito a variações decorrentes das condições ambientas o que pode ocasionar uma falta de padronização do produto além da contaminação por microrganismos. Desta forma, este trabalho objetivou o estudo do comportamento de fécula de mandioca fermentada submetida à secagem por estufa com radiação ultravioleta (UVB e UVC). As propriedades físicoquímicas e tecnológicas avaliadas foram: o conteúdo de umidade final; $\mathrm{pH}$; acidez titulável; absorção de água, expansão e propriedades de pasta (viscosidade). A produção de polvilho azedo por secagem em estufa com UVB mostrou-se promissora, levando aos mesmos resultados de expansão em forno que a fécula fermentada e seca ao sol, possibilitando, ainda, um maior controle de qualidade do produto final.

PALAVRAS-CHAVE: Polvilho azedo; Secagem; Secagem solar; Radiação ultravioleta; amido modificado.
\end{abstract}

ABSTRACT: The production process of sour cassava starch basically consists on natural fermentation of the slurry followed by the sun drying. This process is subjected to the variations of the environment conditions which can result on a lack of the product standard, besides contamination by microorganisms. This way, the present work aims at studying the behavior of fermented cassava starch dried in hothouse employing (UVB and UVC). The physic-chemical and technological properties evaluated were: the final humidity content; $\mathrm{pH}$; titulable acidity; water absorption, expansion and slurry properties (viscosity). The sour cassava starch production by hothouse drying using UVB presented to be interesting, leading to the same expansion in oven as the one fermented and sun dried, makes possible, yet, a greater control on the quality of the final product.

KEYWORDS: Sour cassava starch; Drying; Sun drying; Ultraviolet radiation; Modified starch. 


\section{INTRODUÇÃo}

O polvilho azedo, ingrediente típico da gastronomia brasileira, pode ser utilizado na fabricação de biscoitos, de produtos isentos de glúten e na produção de pão de queijo - produto típico de Minas Gerais.

Consiste de um amido ou fécula de mandioca submetido à fermentação e secagem ao sol, o que resulta em propriedade de expansão sem adição de fermento biológico ou outros agentes químicos (NITSCHKE et al, 2007).

Durante o processo de secagem, toda a fécula fermentada é dispersa em jiraus - uma espécie de suporte feita de bambu, coberta com plástico preto onde o produto permanece por todo o dia, absorvendo calor e radiação solar. $O$ produto é revolvido em intervalos para garantir a secagem homogênea.

A hidrólise resultante do processo fermentativo e da foto oxidação, decorrente dos raios ultravioleta durante a secagem solar resultam em propriedades tecnológicas altamente satisfatórias.

Todos os parâmetros de produção do polvilho azedo são empíricos, tais como o tempo final da fermentação e do processo de secagem. Portanto, o processo não é rigorosamente controlado, resultando em ausência de padronização nos produtos obtidos. Não é estabelecida uma relação entre o tempo e condições de processamento com a qualidade do produto o obtido (MCMINN; MAGEE, 1997).

O processo de secagem ao ar livre sob o sol apresenta muitas desvantagens: os produtos estão expostos à sujeira, poeira e infestação de insetos. Além de apresentar baixas taxas de secagem, a qualidade dos produtos pode ser reduzida devido às condições locais, levando a um subaquecimento ou a um sobreaquecimento e à umidificação pela chuva (ONG, 1996).

Desta forma, com o intuito de ser competitiva no mercado nacional e internacional, toda a cadeia produtiva de polvilho deve buscar melhorias nas características de qualidade e na definição dos parâmetros de produção.

Alguns trabalhos mostraram que a fécula de mandioca tratada com permanganato de potássio e/ou com ácido lático com radiação ultravioleta apresentou um aumento na expansão ao forno, mostrando comportamento similar ao da fécula seca ao sol (NITSCHKE et al., 2007; DEMIATE et al., 1997).

Outros estudos reportaram baixa expansão quando féculas de mandioca foram modificadas por adição de $\mathrm{HCl}$ seguida de secagem solar (PLATA-OVIEDO; CAMARGO, 1998; BERTOLINI et al, 2001a; BERTOLINI et al, 2001b), sugerindo que a estrutura original da fécula e seu nível de hidrólise pode desempenhar papel fundamental durante o processo de expansão.

Bertolini et al. (2001c) e Diniz (2006) reportaram que as radiações ultravioletas decorrentes da secagem solar tem papel fundamental nas propriedades de expansão do polvilho azedo. Entretanto, mudar a maneira de secagem do polvilho azedo não é tão simples devido à necessidade de se ter uma fonte de aquecimento capaz de secar até níveis seguros, estabelecidos pela legislação vigente, e de se ter uma fonte de radiação ultravioleta que deveria ser capaz de fornecer o correto comprimento de onda (BRASIL, 2005).

Por todas as razões apresentadas, este trabalho teve como objetivo principal estudar o efeito da secagem em estufa com lâmpada UVA e UVB nas propriedades físico-químicas e tecnológicas da fécula de mandioca fermentada.

\section{Metodologia}

\subsection{MATÉRIA-PRIMA}

Toda a matéria prima utilizada neste estudo (aproximadamente $6 \mathrm{~kg}$ de fécula de mandioca 
fermentada, proveniente do mesmo tanque de fermentação) foi coletada em uma fábrica localizada na cidade de Formiga, Minas Gerais.

As amostras de fécula de mandioca fermentada no estágio final de fermentação foram separadas em três grupos de teste denominados "seca ao sol", "UVB" e "UVC". O primeiro - "seca ao sol" - foi seco ao sol por aproximadamente 12 horas a partir da fécula fermentada, os grupos - UVB e UVC - foram secos em estufas simples, com circulação forçada de ar, mas utilizando-se uma fonte de radiação ultravioleta instalada no interior desta, como apresentado na FIG. 1. As fontes de radiação ultravioleta emitindo diferentes comprimentos de onda - UVB (280-315 nm) e UVC (100-280 nm) - foram adaptadas dentro da estufa, separadamente. O objetivo era de reproduzir os efeitos da exposição do produto à radiação solar. As amostras foram secas a $60^{\circ} \mathrm{C}$ por 8 horas.

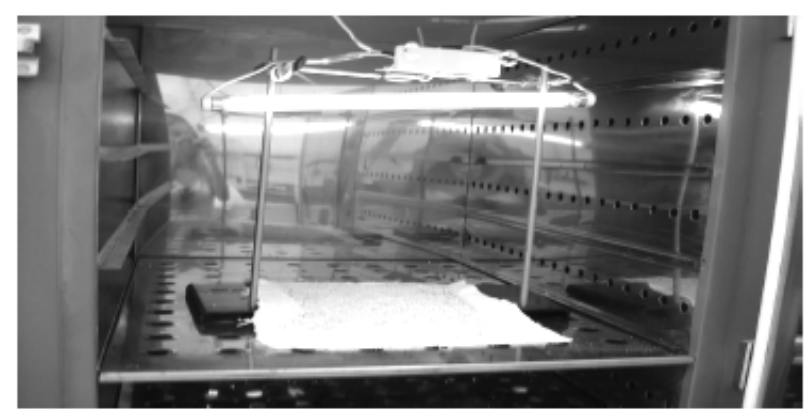

FIGURA 1 - Estufa adaptada com lâmpada UV.

As amostras de polvilho azedo seco em laboratório e seco ao sol foram cominuídas em moinho de martelo para reduzir a granulometria do produto e torná-lo mais adequado às análises laboratoriais.

\subsection{ANÁlises Físico-QUímicas}

As análises físico-químicas realizadas foram: conteúdo de umidade final, acidez titulável, $\mathrm{pH}$ e resíduos. $\mathrm{O}$ conteúdo de umidade foi determinado por meio de secagem em estufa a $105^{\circ} \mathrm{C} / 24$ horas, com circulação de ar, conforme método da Association of Official Analytical Chemistry (AOAC, 1994). As análises de acidez e de $\mathrm{pH}$ foram realizadas de acordo com as Normas Analíticas do Instituto Adolf Lutz (INSTITUTO ADOLF LUTZ, 1976). Os resíduos escuros presentes nas amostras foram determinados de acordo com metodologia descrita em Diniz (2006). Todos os testes foram realizados em triplicata.

\subsection{Propriedades Tecnológicas.}

As propriedades de pasta (viscosidade) foram medidas utilizando-se equipamento Rapid Visco-Analiser (RVA) (Newport Scientific Instruments), (A.A.C.C., 2000). O poder de inchamento foi medido de acordo com procedimento proposto por Cereda (1987). As propriedades de expansão no forno foram determinadas de acordo com metodologia descrita por Maeda e Cereda (2001).

Todos os resultados obtidos foram estatisticamente analisados através de Análise de Variância (ANOVA) complementada com o teste de comparação de médias de Tukey, a um nível de 5\% de significância.

Os experimentos foram conduzidos na Planta Piloto do Centro Universitário de Belo Horizonte - Uni-BH.

\section{Resultados e Discussão}

\subsection{ANÁlises Físico-QUímicAS}

Os dados médios das análises físico-químicas estão apresentados na TAB. 1.

Os resultados mostraram que todas as amostras apresentaram conteúdo de umidade dentro do limite máximo de $18 \%$ estabelecido pela legislação brasileira para fécula de mandioca (BRASIL, 2005). 
De acordo com Machado, Araújo e Pereira. (2010), o baixo $\mathrm{pH}$ em polvilho azedo é considerado uns dos principais responsáveis pelo controle e manutenção de crescimento de microrganismo no polvilho azedo.

Tabela 1

Análises físico-químicas da fécula de mandioca fermentada e seca ao sol ou em estufa (valores médios)

\begin{tabular}{c|c|c|c|c}
\hline $\begin{array}{c}\text { Trata- } \\
\text { mento }\end{array}$ & Amostra * & $\begin{array}{c}\text { Conteúd } \\
\text { o de } \\
\text { umidade* } \\
\text { (\% b.s. })\end{array}$ & $\mathrm{pH}^{*}$ & $\begin{array}{c}\text { Acidez } \\
\text { Titulável } \\
(\mathrm{ml} \\
\mathrm{NaOH} / 100 \\
\mathrm{~g})^{*}\end{array}$ \\
\hline 1 & $\begin{array}{c}\text { Seca ao } \\
\text { Sol } \\
\text { UVB }\end{array}$ & $12,57^{\mathrm{a}}$ & $\begin{array}{c}3,8 \\
\mathrm{a}\end{array}$ & $7,49^{\mathrm{a}}$ \\
3 & UVC & $2,37^{\mathrm{c}}$ & $\begin{array}{c}4,9 \\
\mathrm{a}\end{array}$ & $3,49^{\mathrm{c}}$ \\
\hline $\mathrm{a}$ & $3,24^{\mathrm{c}}$ \\
\hline
\end{tabular}

* Resultados na mesma coluna seguidos pela mesma letra não diferem, estatisticamente, utilizando ANOVA, com nível de $5 \%$ de significância, empregando-se Teste de Tukey;

O polvilho é classificado como doce ou azedo, tendo por base o seu teor de acidez. Para o produto fermentado a acidez deve ser no máximo de $5 \mathrm{~mL}$ de $\mathrm{NaOH} \mathrm{N} / 100 \mathrm{~g}$ e para o não-fermentado de $1 \mathrm{~mL}$ de $\mathrm{NaOH} \mathrm{N} / 100 \mathrm{~g}$ (ABIA.; 2000).

A amostra "Seca ao Sol" apresentou um alto valor de acidez titulável quando comparado com outros grupos. Uma vez que todas as amostras foram obtidas de um mesmo processo de fermentação, estas diferenças são devidas aos processos de secagem. Como a secagem ao sol ocorre de maneira lenta, o processo de fermentação pode ter sido continuado, ainda que de maneira suave, elevando a acidez da amostra. Por outro lado, durante a secagem artificial, pode ter havido perdas de ácidos voláteis, o que levaria a um decréscimo da acidez total média.

Cereda e Vilpoux (2003) sugerem que valores de acidez maiores que 7 caracterizam uma intensa fermentação, enquanto valores menores que 3 caracterizam a ausência de fermentação. Contudo, estes autores consideram que, como trata-se de um produto da fermentação lática, a acidez deve ser considerada como um atributo natural e desejável.

A FIG. 2 apresenta os resultados dos resíduos escuros nas amostras de fécula de mandioca fermentadas e secas ao sol e/ou em estufa com lâmpadas UVB e UVC.

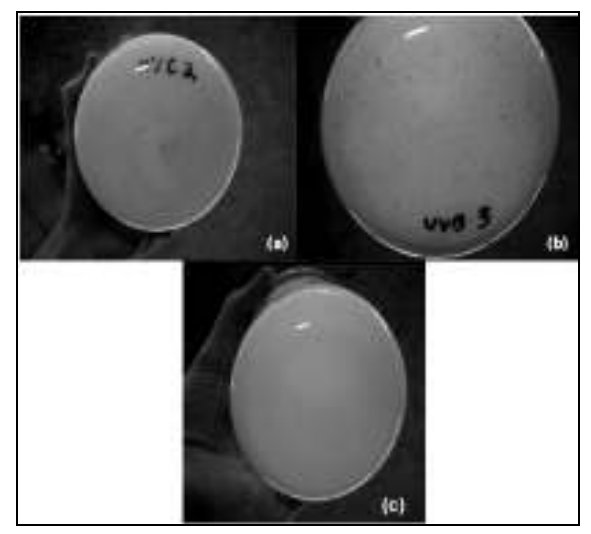

Figura 2 - Resíduos escuros: (a) UVC, (b) UVB e (c) Seco ao Sol

A determinação dos resíduos escuros das amostras de polvilho é útil na avaliação da aceitação industrial ou na rejeição do produto. Um alto número de pontos escuros indica uma purificação insuficiente do polvilho ou um ambiente de secagem inadequado, o que contribui para a contaminação física do produto final. Algumas indústrias estabeleceram um limite máximo de 50 pontos escuros em $50 \mathrm{~g}$ de amostra para sua aceitação como matéria prima. Contudo, no Brasil, não há uma definição na sua legislação.

Os pontos escuros presentes nas amostras UVB e UVC sugerem que a secagem em estufa foi conduzida em uma temperatura alta e/ou por tempo longo, contribuindo para as alterações físicas observadas, tais como grânulos queimados. Njie e Rumsey (1997) realizaram experimentos em um simulador de secagem solar com chips de mandioca e sugeriram que a 
temperatura apresenta o efeito mais significativo na secagem.

\subsection{ANÁlises TECNOLÓgICAS}

As TAB. 2, TAB. 3, TAB. 4 e a FIG. 3 apresentam os resultados das análises tecnológicas para os grupos de amostras - Seca ao Sol, UVB e UVC evidenciando o poder de inchamento, as propriedades de pasta e o índice de expansão no forno da fécula de mandioca fermentada seca ao sol ou em estufa.

O aquecimento na solução aquosa de amido ou fécula leva ao inchamento devido à difusão e absorção de água dentro das regiões amorfas (MAEDA; CEREDA, 2001). A consequência direta deste fenômeno é o aumento da solubilidade, claridade e viscosidade da polpa de fécula. Um incremento da temperatura resulta em mudanças na suspensão aquosa de fécula, aumentando sua capacidade de absorção de água.

Em outras palavras, o poder de inchamento apresenta comportamento dependente da temperatura. Portanto, esta propriedade consiste em uma medida da capacidade de hidratação dos grãos de fécula. Este é um parâmetro importante, uma vez que a qualidade do alimento produzido está frequentemente associada à retenção de água do grão de fécula expandido que é usado como ingrediente. Além disso, através desta característica, é possível obter informação importante sobre a integridade granular da fécula. Danos ao grânulo, por exemplo, pode afetar sua capacidade de absorção de água (DEMIATE; KOTOVICZ, 2011).

Os resultados apresentados na TAB. 2 e na FIG. 3 demonstram que amostras de fécula submetidas a secagem em estufa apresentaram um maior poder de inchamento (absorção de água) a $90^{\circ} \mathrm{C}$ do que aquelas secas ao sol.

Estes dados mostram que, possivelmente, durante este processo de secagem, reações de oxidação ocorreram, permitindo um aumento da disponibilidade dos grupos funcionais (hidroxilas e carboxilas) das moléculas de amilase e de amilopectina, o que acentuaria a interação com a água, além acarretar uma maior viscosidade. Isto ocorre devido à tendência à separação das moléculas oxidadas.

Pode-se observar que a viscosidade da fécula parece ser determinada por fatores como o grau de inchamento dos grãos e sua resistência ao calor e a ação de ácidos e/ou à agitação mecânica.

Tabela 2 -

Poder de inchamento da fécula de mandioca fermentada e seca ao sol ou em estufa

\begin{tabular}{c|c|c|c|c|}
\cline { 2 - 5 } & \multicolumn{4}{|c|}{$\begin{array}{c}\text { Poder de inchamento } \\
\text { (Absorção de água) (g/g) * }\end{array}$} \\
\cline { 2 - 5 } AMOSTRA & $45^{\circ} \mathrm{C}$ & $60^{\circ} \mathrm{C}$ & $75^{\circ} \mathrm{C}$ & $90^{\circ} \mathrm{C}$ \\
\hline $\begin{array}{c}\text { Seca ao } \\
\text { Sol }\end{array}$ & $1,5505^{\mathrm{a}}$ & $6,1777^{\mathrm{b}}$ & $12,7326^{\mathrm{a}}$ & $4,0757^{\mathrm{b}}$ \\
UVB & $2,578^{\mathrm{a}}$ & $7,0164^{\mathrm{a}}$ & $13,636^{\mathrm{a}}$ & $7,5820^{\mathrm{a}}$ \\
UVC & $2,7355^{\mathrm{a}}$ & $6,7823^{\mathrm{ab}}$ & $13,2292^{\mathrm{a}}$ & $7,5238^{\mathrm{a}}$ \\
\hline
\end{tabular}

(*) Os resultados das médias acompanhados da(s) mesmas letras na mesma coluna não são estatisticamente diferentes, a um nível de $5 \%$ de significância, pelo teste de Tukey.

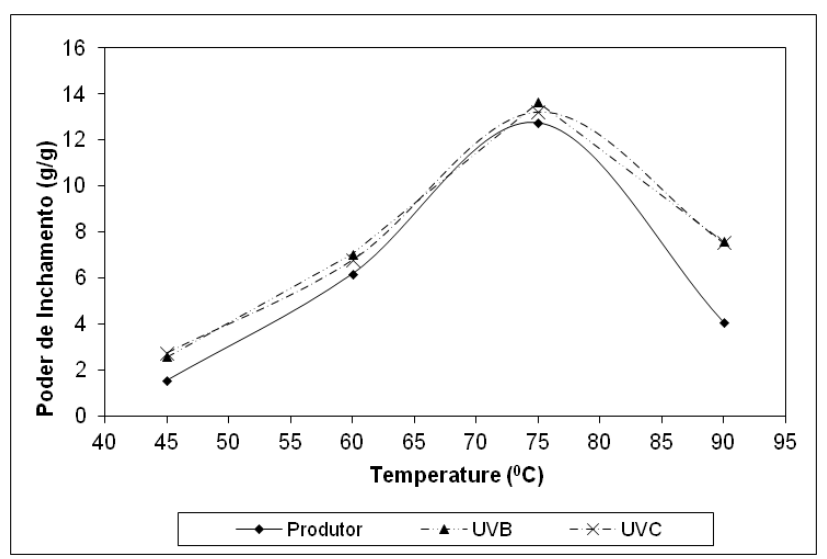

Figura 3 - Perfis de poder de inchamento nas temperaturas de $45,60,75$ e $90^{\circ} \mathrm{C}$. 
Tabela 3

Propriedades de pasta (viscosidade) da fécula de mandioca fermentada e seca ao sol ou em estufa

\begin{tabular}{c|c|c|c}
\cline { 2 - 4 } AMOSTRA $^{*}$ & \multicolumn{3}{|c}{ Viscosidade } \\
\cline { 2 - 4 } & $\begin{array}{c}\text { Viscosidade } \\
65^{\circ} \mathrm{C} \\
(\mathrm{RVU})\end{array}$ & $\begin{array}{c}\text { Viscosidade } \\
75^{\circ} \mathrm{C} \\
(\mathrm{RVU})\end{array}$ & $\begin{array}{c}\text { Viscosidade } \\
\text { Final } \\
90^{\circ} \mathrm{C} \\
(\mathrm{RVU})\end{array}$ \\
\hline Seca ao Sol & $625^{\mathrm{a}}$ & $575^{\mathrm{a}}$ & $475^{\mathrm{a}}$ \\
UVB & $725^{\mathrm{b}}$ & $730^{\mathrm{b}}$ & $675^{\mathrm{b}}$ \\
UVC & $770^{\mathrm{b}}$ & $770^{\mathrm{b}}$ & $690^{\mathrm{b}}$ \\
\hline
\end{tabular}

(*) Os resultados das médias acompanhados da(s) mesmas letras não são estatisticamente diferentes, a um nível de $5 \%$ de significância, pelo teste de Tukey.

Tabela 4

Propriedades de expansão da fécula de mandioca fermentada e seca ao sol ou em estufa

\begin{tabular}{c|c|c|c}
\hline AMOSTRA $^{*}$ & $\begin{array}{c}\text { Seca } \\
\text { ao Sol }\end{array}$ & UVB & UVC \\
$\begin{array}{c}\text { Expansão ao } \\
\text { forno (ml/g) }\end{array}$ & $4,768^{a}$ & $3,242^{a b}$ & $2,734^{b}$ \\
\hline
\end{tabular}

$\left(^{*}\right)$ Os resultados das médias acompanhados da(s) mesmas letras não são estatisticamente diferentes, a um nível de $5 \%$ de significância, pelo teste de Tukey.

De acordo com os dados da TAB. 3 observa-se que todos os grupos - Seco ao Sol, UVA e UVB apresentaram dados de viscosidade típicos de amidos fermentados, com um abrupto incremento da consistência até atingir o pico de viscosidade máxima. Esse comportamento, de acordo com Machado, Araújo e Pereira (2010) sugere a existência de forcas de associação fracas na manutenção da estrutura do granulo deste tipo de amido.

A viscosidade mais alta da fécula fermentada e seca artificialmente condiz com os resultados obtidos no que se refere à capacidade de absorção de água (TAB. 2) e pode ser devida à presença de moléculas oxidadas.
Além disto, o maior grau de acidez da amostra Seca ao Sol poderia provocar modificações estruturais no amido, como, por exemplo, a hidrólise de moléculas de amilose e/ou amilopectina, prejudicando assim o aumento da viscosidade.

Deste modo, estes resultados sugerem que amostras submetidas à secagem por radiação artificial (UVB e UVC) têm melhor capacidade de inchamento (retenção de água) e maior viscosidade que a amostra seca ao sol.

A avaliação da expansão ao forno para o polvilho azedo é realizada através de um teste, adotado por usuários e produtores, para verificação de sua qualidade. De acordo com Nunes (1997), a expansão ao forno é considerada pequena quando o valor apresentado é inferior a $5,0 \mathrm{ml} / \mathrm{g}$, média quando este valor está compreendido entre 5,0 e $10,0 \mathrm{ml} / \mathrm{g}$, e grande para valores maiores que $10 \mathrm{ml} / \mathrm{g}$. Maeda e Cereda (2001) propuseram outro tipo de classificação para o polvilho azedo: tipo $A$, para polvilho que apresenta expansão ao forno acima de $16 \mathrm{ml} / \mathrm{g}$; tipo $\mathrm{B}$, para expansão ao forno entre 12,0 e $16,0 \mathrm{ml} / \mathrm{g}$ e tipo $\mathrm{C}$, para polvilho com expansão ao forno menor que 12 $\mathrm{ml} / \mathrm{g}$.

A TAB. 4 e a FIG. 4 demonstram que tanto as amostras secas ao sol quanto as secas em estufa apresentaram baixo índice de expansão e podem ser classificados como tipo $\mathrm{C}$, possivelmente devido ao processo de fermentação que é responsável por modificações no grânulo pelos ácidos produzidos, levando a um aumento da capacidade de solubilização e intumescimento em água nos polvilhos azedos.

De acordo com Cereda (1987), a água, que o grânulo pode absorver e reter, é um fator de qualidade importante, pois está relacionada com o poder de expansão da massa do polvilho azedo ao forno. Desta forma, devido aos resultados obtidos em relação às propriedades de pasta e a absorção de água seria 
esperado que os grupos UVB e UVC apresentassem uma maior expansão que o grupo Seco ao Sol.

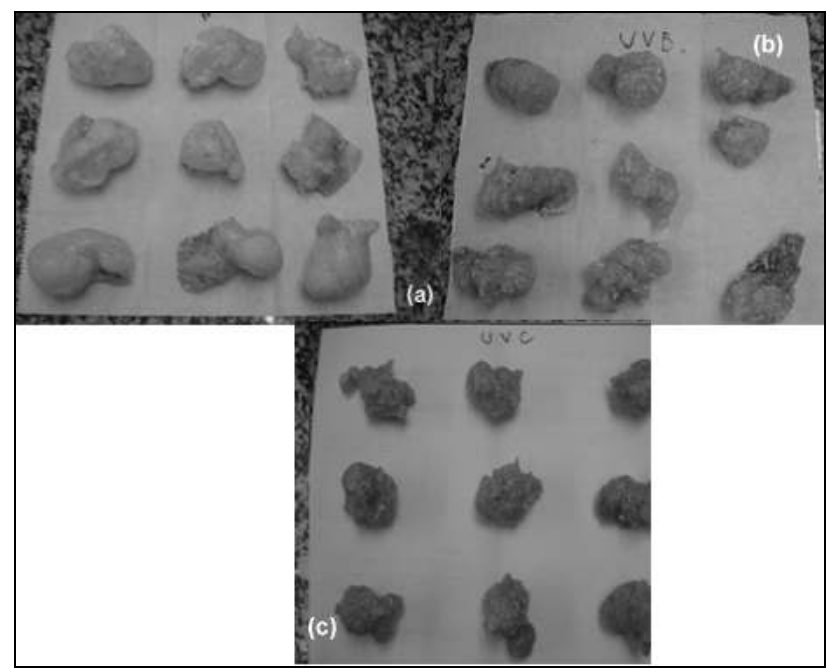

Figura 4 - Biscoitos feitos com polvilho azedo:

(a) Seco ao sol; (b) Sem lâmpada; (c) UVB e (d) UVC.

Entretanto, a baixa umidade inicial, as condições de secagem mais severas, comprovadas pela quantidade de pontos pretos presentes neste grupo (Figura 2), e o tipo de radiação empregado podem ter prejudicado a propriedade de expansão do grupo UVC. O grupo UVB apresentou propriedades de expansão semelhantes ao grupo Seco ao Sol. Sendo assim, os resultados deste trabalho mostraram que a secagem em estufa com lâmpada UVB foi eficiente na substituição da secagem solar.

\section{CONCLUSÕES}

As féculas fermentadas e secas ao sol (polvilho azedo) ou secas por estufa com lâmpada UVB e UVC foram classificadas como polvilho tipo $\mathrm{C}$ devido à expansão ao forno menor que $12 \mathrm{ml} / \mathrm{g}$. Esta baixa expansão pode ser atribuída à fermentação que é natural, sem condições de controle.

O grupo UVC apresentou menor expansão que o grupo Seco ao Sol, possivelmente devido às condições mais severas de secagem. Desta forma, novos estudos devem ser conduzidos em condições de secagem mais suaves.

A secagem por UVB, por outro lado, se mostrou eficiente, uma vez que não houve diferença significativa em relação às propriedades de expansão.

Sendo assim, conclui-se que a secagem artificial com radiação UVB pode ser utilizada, apresentando-se como técnica promissora para a substituição da secagem solar, garantindo um maior controle e higiene dos produtos.

\section{Agradecimentos}

Os autores agradecem ao Centro Universitário de Belo Horizonte - UniBH pela disponibilização da Planta Piloto para realização dos ensaios.

\section{REFERÊNCIAS}

A.A.C.C. American Association of Cereal Chemists (2000). Approved methods of AACC, 10th ed, Method 61-02. The Association: St. Paul. MN.

A.O.A.C. (Association of Official Analytical Chemistry).(1994), Official methods of analysis. $14 \mathrm{ed}$. Arlington: $1141 \mathrm{p}$.
ASSOCIACAO BRASILEIRA DAS INDUSTRIAS DE ALIMENTOS (ABIA). Compêndio da legislação de alimentos: atos do Ministério da Saúde. São Paulo, 2000. Não paginado.

BERTOLINI, A. C. ; MESTRES, C. ; COLONNA, P. ; RAFFI, J. Free radicals formation in the UV and 
gamma irradiated cassava starch.. Carbohydrate Polymers, v. 44, n. 2, p. 269-271b, 2001.

BERTOLINI, A. C; MESTRES, C.; RAFFI, J. BULÉON, A.; LERNER, D.; COLONNA, P. . Photodegradation of cassava and corn starches. Journal Agricultural Food Chemistry, v. 49 (2), p. 675-682a, 2001.

BRASIL. Resolução RDC ANVISA/MS n. 263/05, de 22 de setembro de 2005. Regulamento Técnico para Produtos de Cereais, Amidos, Farinhas e Farelos. Diário Oficial da União, Brasília, DF, 23 dez. 2005.

CEREDA, M.P. Tecnologia e qualidade do Polvilho azedo. Informe Agropecuário. Belo Horizonte, v. 13, n. 145, p. 63-68, 1987.

CEREDA, M.P.; VILPOUX, O.F. Culturas de Tuberosas Amiláceas Latino Americanas:Tecnologia, usos e potencialidades de tuberosas amiláceas Latino Americanas. v.3,cap.13 Fundação Cargill - São Paulo, 2003.

DEMIATE, I. M.; KOTOVICZ, V. Cassava starch in the Brazilian food industry. Ciência e Tecnologia de Alimentos, Campinas, 31(2): 388-397, abr.-jun. 2011.

DEMIATE, I. M. et al. Characteristics of quality of sour starch samples produced or commercialized in Paraná State.Arquivos de Biologia e Tecnologia, Curitiba, 40(2):321-330, 1997.

DINIZ, I.P. Caracterização tecnológica do polvilho azedo produzido em diferentes regiões do estado de Minas Gerais. 2006. 101f. Dissertação (Mestrado em Ciência e Tecnologia de Alimentos) - Faculdade de Engenharia de Alimentos, Universidade Federal de Viçosa (UFV), Viçosa, 2006.

INSTITUTO ADOLFO LUTZ. Normas Analíticas do Instituto Adolfo Lutz. São Paulo, SP: Secretaria do Estado da Saúde, v.1, 371p, 1976.

MACHADO, A. V.; ARAÚJO, F. M. M. C. de; PEREIRA, J. . Caracterização física, química e tecnológica do polvilho azedo. Revista Verde de Agroecologia e Desenvolvimento Sustentável, v. 5, p. 01-06, 2010.

MAEDA, K.C.; CEREDA, M.P. Avaliação de duas metodologias de expansão ao forno do polvilho azedo. Ciência e Tecnologia de Alimentos, Campinas, v.21, n.2, p.139-143, mai/ago, 2001.

MCMINN, W.A.M.; MAGEE, T.R.A. Quality and physical structure of a dehydrated starch-based system Drying Technology, v.15, issue 6-8, p.19611971.

NIJIE, D.N.; RUMSEY, T.R. Influence of factors on the drying of cassava in a solar simulator Drying Technology, v.15, issue3-4, p.1187-1202, 1997.

NITSCHKE, M.; BERTOLINI, A. C., GARCIA, C. F., TEIXEIRA, M. A. V. Biopolymers on Food Technology: Produciton and Applications of Modified Starches. In: Andréa C. Bertolini. (Org.). Biopolymers Tecnology. São Paulo: Cultura Acadêmica, p. 13-44, 2007.

NUNES, O. L. G. S. Efeito da radiação ultravioleta sobre as propriedades funcionais da fécula de mandioca tratada com ácido lático. 1994. 88 f. Dissertação (Mestrado em Agronomia - Energia na Agricultura) - Faculdade de Ciências Agronômicas, Universidade Estadual Paulista "Júlio de Mesquita Filho", Botucatu, 1994ABNT - NBR 6022: Informação e documentação - Artigo em publicação periódica científica impressa - Apresentação. Rio de Janeiro, maio 2003. 5 p.

ONG, K.S. Experimental investigation of a solar bamboo dryer. Drying Technology, v.14, issue 10, p.2411-2417, 1996.

PLATA-OVIEDO, M. S. V.; CAMARGO, C. Effect of Acid Treatments and Drying Processes on Physicochemical and Functional Properties of Cassava Starch. Journal of the Science of Food and Agriculture, v.77(1), p.103-108, 1998. 